\title{
Contextos y creatividad Variables sociodemográficas y datos normativos en el Test CREA
}

\author{
Contexts and Creativity \\ Sociodemographic Variables and Normative Data in the CREA Test
}

\author{
Romina Elisondo * 1, Danilo Donolo ${ }^{1}$ \\ 1 - Consejo Nacional de Investigaciones Científicas y Técnicas. Departamento de Ciencias de la Educación. \\ Facultad de Ciencias Humanas. Universidad Nacional de Río Cuarto. Argentina.
}

Recibido: 29/04/2018 Revisado: 19/06/2018 Aceptado: 07/07/2018

Resumen

El Test CREA es un instrumento de evaluación de la creatividad que valora la capacidad de las personas de formular interrogantes ante determinados estímulos. En el presente estudio se analizan desempeños en el Test CREA de niños, adolescentes y adultos que residen en la provincia de Córdoba (Argentina). Se utilizó el Test CREA para medir la creatividad por sus propiedades psicométricas. Al grupo de estudio lo constituyen 3237 niños, adolescentes y adultos. Las variables de incidencia analizadas fueron género, edad y escolaridad. Los resultados indican diferencias significativas según edad en los tres grupos estudiados, según género en adolescentes y según escolaridad en adultos. En el artículo se proponen datos normativos que atienden a las diferencias observadas. Los datos presentados son de relevancia para la interpretación de los resultados del Test CREA en grupos y contextos diversos.

Palabras clave: creatividad, test CREA, normas, género, edad, escolaridad

\begin{abstract}
The CREA Test is an instrument for evaluating creativity that assesses the ability of people to ask questions about certain stimuli. In this study we analyze the performances of the CREA Test of children, adolescents and adults residing in the province of Córdoba (Argentina). The CREA Test was used to measure creativity for its proven technical characteristics. The study group consists of 3237 children, adolescents and adults; the incidence variables analyzed were gender, age and schooling. The results indicate significant differences regarding age in the three groups studied, regarding gender in adolescents and regarding schooling in adults. The article proposes normative data that address the differences observed. The presented data are relevant for the interpretation of the results of the CREA Test in different groups and contexts.
\end{abstract}

Key words: creativity, CREA test, norms, gender, age, education

\footnotetext{
* Correspondencia a: Romina Elisondo. Departamento de Ciencias de la Educación. Facultad de Ciencias Humanas. Universidad Nacional de Río Cuarto. Ruta 36 Km 601. Río Cuarto CP 5800 Córdoba Argentina. relisondo@gmail.com 00543584676473 


\section{Introducción}

En la bibliografía especializada, generalmente se acepta que todas las personas son creativas en algún sentido y en alguna actividad de su vida (Richards, 2007). En nuestros trabajos anteriores también hemos adherido a esta idea promisoria y alentadora para dar más espacio a la educación y al aprendizaje. Nuestro interés es analizar cómo se desempeña en una prueba de creatividad un grupo de sujetos en distintas etapas de su desarrollo y con diferentes niveles de escolaridad. El objetivo es contar con los elementos para sugerir acciones que tiendan a que la mayoría de las personas pueda encontrar un ambiente en el que expresar sus capacidades creativas.

La complejidad del constructo definido como creatividad dificulta contar con consensos amplios en cuanto a los alcances y los límites de su definición y a los acuerdos para su medición. Por eso, surgen inquietudes entre los investigadores respecto a la posibilidad de medir la creatividad, los aspectos o dominios que podrían evaluarse y los indicadores más apropiados.

Estas son algunas de las difíciles preguntas que intentan responder, sin pocas controversias y debates, los investigadores interesados en la medición de la creatividad (Baer, 2012; Batey, 2012; Barbot, Besançon, \& Lubart, 2016). Desde los estudios psicométricos iniciales de Guilford (1962), hasta la actualidad, se han creado, perfeccionado y estandarizado diferentes instrumentos para evaluar la creatividad y sus diversas manifestaciones. La mayoría de los instrumentos estandarizados se orientan a valorar capacidades creativas a partir de tareas y procedimientos que evalúan el pensamiento divergente. El Test de Pensamiento Creativo de Torrance es uno de los instrumentos más utilizados en la intervención e investigación psicológica (Humble, Dixon, \& Mpofu, 2017; Torrance, 1990). Se han realizado numerosos estudios de validación y estandarización del instrumento en diversos lugares geográficos, como por ejemplo en Turquía, Corea, Portugal y Brasil (Primi, Nakano, Morais, Silva-Almeida, \& Marques-David, 2013; Yarbrough, 2016; Yoon, 2017; Wechsler, 2006) y también en Argentina (Aranguren, 2014; Krumm, Aranguren, Arán-Filippetti, \& Lemos, 2016). También se han desarrollado instrumentos estandarizados para evaluar logros creativos en diferentes campos del conocimiento como el Creative Behaviors Inventory (Hocevar, 1979), el Creative Achievement Questionnaire (Carson, Peterson, \& Higgins, 2005) y el Biographical Inventory of Creative Behaviors (Batey, 2007). En Argentina se han confeccionado dos cuestionarios orientados a valorar comportamientos y acciones creativas en diferentes situaciones y áreas del conocimiento: la Escala de Comportamiento Creativo (Aranguren \& Irrazabal, 2012) y el Cuestionario de Acciones Creativas (Elisondo \& Donolo, 2016a).

Los estudios psicométricos son los más desarrollados en el campo de la creatividad, tal como lo afirman Long (2014) y Said-Metwaly, Van den Noortgate y Kyndt (2017) a partir del análisis de artículos de investigación publicados en revistas especializadas. Said-Metwaly et al. (2017) mencionan limitaciones en los instrumentos, específicamente aquellas vinculadas con la falta de validez y la valoración de aspectos individuales sin considerar variables contextuales. Asimismo, sugieren analizar las posibilidades de integración de diferentes instrumentos y ponen énfasis en la importancia de considerar la variabilidad de los perfiles de creatividad en contextos, dominios y etapas evolutivas diferentes.

Considerando la relevancia de disponer de datos normativos actualizados que atiendan a diferencias sociodemográficas, el presente artículo se dispone a analizar la incidencia del género, la edad y el nivel de escolaridad en las puntuaciones 
en el Test CREA (Corbalán-Berná, Martínez-Zaragoza, Donolo, Alonso-Monreal, Tejerina-Arreal y Limiñana- Gras, 2003) y presentar datos normativos para tres etapas etarias. El Test CREA evalúa la inteligencia creativa en niños, adolescentes y adultos a partir de una tarea general: la formulación de interrogantes ante estímulos visuales. El Test CREA se fundamenta teóricamente en las perspectivas teóricas de la creatividad que enfatizan el valor de los procesos de formulación de problemas. Desde estas perspectivas, se considera que la formulación de interrogantes es el proceso cognitivo básico de la creatividad. A partir de los procesos de formulación de problemas emergen ideas y productos creativos. En esta línea argumentativa, la creatividad es definida como la capacidad de formular cuestiones y resolver problemas de manera divergente (Corbalán-Berná, 2008).

El Test CREA ha sido utilizado en numerosas investigaciones en diversos países y con variadas poblaciones (Corbalán-Berná et al., 2003; Corbalán-Berná et al., 2014; López-Martínez \& Navarro-Lozano, 2008). Se han realizado estudios de las propiedades psicométricas del instrumento en poblaciones española, argentina y estadounidense (Clapham \& King, 2010; Martínez-Zaragoza, 2003), se hallaron índices de confiabilidad adecuados y se mostraron diversas evidencias de validez. Se han observado correlaciones entre capacidades creativas medidas con el CREA y las mediciones realizadas con la Batería de Guilford y el Test de Torrance (Corbalán-Berná et al., 2003; Clapham \& King, 2010; López-Martínez \& Navarro-Lozano, 2008). El Test CREA es un instrumento utilizado extensamente en la investigación de la creatividad, tal como se visualiza en investigaciones actuales con niños (Antoñanzas-Laborda, Toner-Pelay, Salavera-Bordás, Soler-Costa, \& Usan-Supervía, 2015; Cárdenas-Avila, López-Fernández, \& Arias-Cas- tro, 2018), adolescentes (Castañeda-Rey, López, \& Ezquerro-Cordón, 2017; Ramos-Moreno, López-Fernández, \& Llamas-Salguero, 2017; Sánchez-Hernández, Méndez, \& Garber, 2015) y adultos (Bogaert-García, 2017; RodríguezSuárez, Llamas-Salguero, \& López-Fernández, 2015).

En Argentina se han realizado diferentes estudios del Test CREA: análisis de relaciones entre creatividad, inteligencia (Elisondo \& Donolo, 2010) y personalidad (Elisondo, Donolo, \& Corbalán-Berná, 2009), pruebas exploratorias con nuevos estímulos (Elisondo \& Donolo, 2011), estudios de correlaciones entre creatividad verbal y gráfica (Freiberg-Hoffmann \& Fernández-Liporace, 2017) e indagación de relaciones entre capacidades creativas y participación en actividades de ocio (Chiecher, Elisondo, Paoloni, \& Donolo, 2018; Elisondo \& Donolo, 2014; 2016a; 2016b). También se han realizado estudios exploratorios de diferentes procedimientos de puntuación de las preguntas (Elisondo, 2015) y análisis de la originalidad de los interrogantes (Elisondo, Donolo, \& Limiñana-Gras, 2018).

El objetivo principal de esta investigación es analizar los desempeños en el CREA de una muestra amplia de niños, adolescentes y adultos argentinos de diferentes edades y niveles de escolaridad. En los tres grupos etarios, se analiza la incidencia del género, la edad y la escolaridad en el Test CREA y se presentan datos normativos que atienden a las particularidades de los grupos estudiados. Se considera de relevancia disponer de datos actualizados y referencias normativas sobre el instrumento que consideren particularidades sociodemográficas teniendo en cuenta que en estudios anteriores se han observado diferencias según género, edad y escolaridad de los participantes (Chacón-Araya \& Moncada-Jiménez, 2006; Donolo \& Elisondo, 2007; Elisondo \& Donolo 2011; Limiñana-Gras, Bordoy, Juste-Ballesta, \& 
Corbalán-Berná, 2010). La relevancia del estudio presentado también se vincula con la amplitud de la muestra seleccionada $(\mathrm{N}=3237)$ y la diversidad de casos incluidos (varones y mujeres de diferentes edades y niveles de escolaridad). La significatividad del estudio también se vincula con los desafíos actuales de la psicometría argentina y la necesidad de contar con estudios psicométricos y datos normativos para el uso de instrumentos de medición en contextos locales (Contini, 2013).

\section{Método}

Participantes

La muestra estuvo conformada por 3237 personas que residían al momento de la investigación en la provincia de Córdoba (Argentina). Participaron 192 niñas y 205 niños (entre 6 y 11 años), 421 adolescentes mujeres y 299 varones (entre 12 y 17 años) y 1352 mujeres y 768 hombres adultos (entre 18 y 82 años). Se incluyeron en la muestra jóvenes y adultos con diferentes experiencias educativas, según los tres niveles del Sistema Educativo Argentino: primario (8\%), secundario $(30 \%)$ y superior $(62 \%)$. Las niñas y los niños de la muestra cursaban el nivel primario y los adolescentes el nivel secundario de la educación obligatoria argentina, en escuelas de gestión pública y privada. Todos los participantes eran de nacionalidad argentina y nivel socioeconómico medio.

\section{Instrumentos}

Se utilizaron como instrumentos de recolección de datos el Test CREA y un cuestionario breve sobre datos sociodemográficos. El cuestionario recoge datos acerca del género, la edad, los estudios en curso o el nivel de escolaridad alcanzado (en el caso de quienes ya no se encuentran en el sistema educativo) y el lugar de residencia de los participantes.

El objetivo del Test CREA es medir la inteligencia creativa a partir de la evaluación de la capacidad de las personas de formular interrogantes ante estímulos visuales. Tres láminas (A, B y C) conforman el instrumento. La consigna del test es: Se te va a presentar una ilustración. Tu tarea consiste en escribir brevemente todas las preguntas que puedas hacer sobre lo que representa la lámina. Trata de hacer el mayor número posible de preguntas en cuatro minutos. La aplicación del test es escrita, individual o colectiva, a individuos mayores de 10 años. Entre los $6 \mathrm{y}$ los 9 años la administración debe ser individual y oral. Las propiedades psicométricas del instrumento han sido documentadas a partir de investigaciones en diferentes contextos geográficos (Clapham \& King, 2010; Corbalán-Berná et al., 2014; Corbalán-Berná et al., 2003; López-Martínez \& Navarro-Lozano, 2008; López-Martínez \& Brufau, 2010; Martínez-Zaragoza, 2003). En el manual del test se detallan estudios de fiabilidad y validez, se analizan formas paralelas (.87) y se presentan evidencias de validez convergente con la Batería de Guilford $(r=.61 ; r=.75 ; r=.59 ; r$ $=.48) \mathrm{y}$ de validez discriminante con pruebas de inteligencia $(r=.25 ; r=.20)$. Considerando criterios externos, también se observan correlaciones moderadas entre el CREA y las puntuaciones de expertos en una tarea creativa (Corbalán-Berná et al., 2003).

\section{Análisis de datos}

Se realizaron análisis mediante el programa especializado de estadísticas para ciencias sociales y humanas SPSS. Se realizaron estudios de frecuencia, media, desviación estándar, mínimo y máximo en las tres láminas y en los tres grupos. 
Con el objetivo de analizar la incidencia de variables sociodemográficas (género, edad y escolaridad), se realizaron estudios de diferencia de media mediante prueba $t$ y ANOVA. Para la construcción de datos normativos y la obtención de puntuaciones derivadas se calcularon percentiles para cada lámina y grupo. En el presente estudio, al igual que en el manual del test, se realizaron transformaciones no lineales para la construcción de baremos. De esta manera, es posible comparar los baremos disponibles en el manual del test con los datos normativos propuestos en la presente investigación. Los grupos considerados también son similares a los definidos en los baremos del manual del CREA.

\section{Resultados}

Niños

Los análisis descriptivos del presente estudio (Lámina $\mathrm{A}: \mathrm{M}=10.86, \mathrm{DE}=5.53$; Lámina $\mathrm{B}: \mathrm{M}=11.48, \mathrm{DE}=7.18$; Lámina $\mathrm{C}: \mathrm{M}=8.02$, $\mathrm{DE}=5.25)$ indican puntajes medios superiores a los establecidos en el manual del Test CREA para la población argentina de 6 a 11 años (Lámina A: $\mathrm{M}=5.48, \mathrm{DE}=3.78$; Lámina $\mathrm{C}: \mathrm{M}=4.22, \mathrm{DE}$ $=3.47$ ). Con respecto a la incidencia del género en el CREA, no se observan diferencias estadísticamente significativas entre los puntajes de los niños y los de las niñas (ver Tabla 1).

Tabla 1

Media y prueba $t$ en las tres láminas del CREA según género de los niños.

\begin{tabular}{ccccc}
\hline Género & & CREA A & CREA B & CREA C \\
\hline Niñas & $\mathrm{M}$ & 10.94 & 11.19 & 8.38 \\
& $\mathrm{DE}$ & 5.35 & 7.35 & 4.99 \\
Niños & $\mathrm{M}$ & 10.80 & 11.74 & 7.65 \\
& $\mathrm{DE}$ & & 7.07 & 5.50 \\
& & & $t_{(1,314)}=1.241$ \\
& & $t_{(1,313)}=.232$ & $t_{(1,141)}=-.460$ & .215 \\
\hline
\end{tabular}

La prueba ANOVA indica diferencias estadísticamente significativas en el CREA según la edad de los participantes (ver Tabla 2). A partir del análisis exploratorio de las medias se decidió conformar dos grupos que muestran diferencias en los desempeños en el CREA: el primero conformado por niños de 6 a 8 años y el segundo por niños de 9 a 11 años. Se observaron diferencias de medias estadísticamente significativas entre ambos grupos en el CREA (ver Tabla 3).
Se realizaron contrastes post hoc y se observaron diferencias significativas en la Lámina A entre las medias de niños de 6,7 y 8 años y la medias de niños de los niños de 9, 10 y 11 años. Entre los niños de 9, 10 y 11 años no se observaron diferencias significativas de media en la Lámina A. En la Lámina B se observaron diferencias entre los niños de 11 años y los de 6, 7, 8, 9 y 10 años. En la Lámina $\mathrm{C}$, se observaron diferencias entre los de 6 y los de 7, 8, 9, 10 y 11 años, entre los de 7, 9 y 10 años, entre los de 8, 9 y 10 años y entre los de 10 y 11 años. 
Tabla 2

Media y prueba ANOVA en las tres láminas del CREA según edad de los niños.

\begin{tabular}{|c|c|c|c|c|}
\hline Edad & & CREA A & CREA B & CREA C \\
\hline \multirow{2}{*}{$\begin{array}{c}6 \text { años } \\
(n=44)\end{array}$} & M & 3.09 & 3.55 & 2.40 \\
\hline & $\mathrm{DE}$ & 2.91 & 3.24 & 3.24 \\
\hline \multirow{2}{*}{$\begin{array}{l}7 \text { años } \\
(\mathrm{n}=50)\end{array}$} & M & 4.15 & 5.50 & 6.32 \\
\hline & $\mathrm{DE}$ & 2.91 & 2.99 & 3.79 \\
\hline \multirow{2}{*}{$\begin{array}{l}8 \text { años } \\
(n=27)\end{array}$} & M & 5.70 & 6.37 & 6.25 \\
\hline & $\mathrm{DE}$ & 1.92 & 3.20 & 3.85 \\
\hline \multirow{2}{*}{$\begin{array}{c}9 \text { años } \\
(n=82)\end{array}$} & M & 10.73 & 6.22 & 8.39 \\
\hline & $\mathrm{DE}$ & 4.53 & 3.11 & 3.85 \\
\hline \multirow{2}{*}{$\begin{array}{l}10 \text { años } \\
(\mathrm{n}=87)\end{array}$} & M & 12.50 & 7.16 & 12.29 \\
\hline & $\mathrm{DE}$ & 5.41 & 3.18 & 5.31 \\
\hline \multirow{3}{*}{$\begin{array}{c}11 \text { años } \\
(\mathrm{n}=107)\end{array}$} & M & 12.30 & 14.55 & 7.19 \\
\hline & $\mathrm{DE}$ & 5.38 & 6.84 & 3.41 \\
\hline & & $\mathrm{F}_{(5,309)}=18.65^{* *}$ & $\mathrm{~F}_{(5,137)}=14.88^{* *}$ & $\mathrm{~F}_{(5,310)}=36.66^{* *}$ \\
\hline
\end{tabular}

Nota. ${ }^{* *} p<.01$

Tabla 3

Media y prueba $t$ en las tres láminas del CREA según dos grupos de edades: 6-8 años y 9-11 años.

\begin{tabular}{ccccc}
\hline Edad & & CREA A & CREA B & CREA C \\
\hline 6-8 años & M & 4.59 & 5.40 & 4.88 \\
& DE & 2.67 & 3.27 & 4.05 \\
$9-11$ años & M & 11.88 & 13.45 & 9.97 \\
& DE & 5.19 & 7.00 & 4.97 \\
& & & $t_{(1.141)}=-6.55^{* *}$ & $t_{(1.314)}=-9.47^{* *}$ \\
\hline
\end{tabular}

Nota. ${ }^{* *} p<.01$ 
Conforme a las diferencias observadas según $e d a d$, se considera pertinente presentar datos normativos para cada uno de los grupos de niños (de 6 a 8 años y de 9 a 11 años). Se proponen a continuación percentiles para cada una de las láminas del CREA en los dos grupos de niños definidos según edad (ver Tabla 4).

Tabla 4

Percentiles para niños de 6 a 8 y de 9 a 11 años en las Láminas A, B y C del Test CREA.

\begin{tabular}{ccccccc}
\hline & \multicolumn{1}{c}{ Niños de 6 a 8 años } & \multicolumn{3}{c}{ Niños de 9 a 11 años } \\
\hline Percentil & A & B & C & A & B & C \\
95 & 9 & 11 & 13 & 13 & 11 & 11 \\
90 & 8 & 9 & 8 & 11 & 10 & 10 \\
75 & 7 & 7 & 7 & 9 & 8 & 8 \\
50 & 5 & 6 & 5 & 7 & 7 & 6 \\
25 & 3 & 3 & 4 & 5 & 5 & 5 \\
10 & 1 & 1 & 1 & 3 & 3 & 3 \\
5 & & & & 1 & 1 & 1 \\
$\mathrm{~N}$ & 44 & 35 & 195 & 271 & 108 & 195 \\
$\mathrm{M}$ & 4.59 & 5.40 & 4.87 & 11.88 & 13.45 & 9.97 \\
$\mathrm{DE}$ & 2.67 & 3.27 & 4.05 & 5.19 & 7.00 & 4.97 \\
\hline
\end{tabular}

\section{Adolescentes}

Los puntajes medios observados en el presente estudio (Lámina $\mathrm{A}: \mathrm{M}=11, \mathrm{DE}=4.79$; Lámina $\mathrm{B}: \mathrm{M}=10.84, \mathrm{DE}=5.10$; Lámina $\mathrm{C}: \mathrm{M}$ $=10.17, \mathrm{DE}=5.44)$ son similares a los establecidos en el manual del Test CREA para la Lámina C $(\mathrm{M}=10.66, \mathrm{DE}=5.8)$ e inferiores en la Lámina A $(\mathrm{M}=12.86, \mathrm{DE}=5.8$; ver Tabla 10). Al analizar las variaciones según género, se observan diferencias estadísticamente significativas a favor de las adolescentes en las Láminas A y B del CREA (ver Tabla 5).

El ANOVA mostró diferencias estadísticamente significativas en las tres láminas del CREA según la edad de los participantes (ver Tabla 6). Al igual que en la muestra de niños, se decidió conformar dos grupos según edad: de 12 a 14 años y de 15 a 17 años. Se observaron diferencias de medias estadísticamente significativas entre ambos grupos en las Láminas A y B del CREA (ver Tabla 7).

Tabla 5

Media y prueba $t$ en las tres láminas del CREA según género en adolescentes.

\begin{tabular}{ccccc}
\hline Género & & CREA A & CREA B & CREA C \\
\hline $\mathrm{M}$ & 11.32 & 11.45 & 10.34 & 5.57 \\
& $\mathrm{DE}$ & 4.89 & 5.31 & 9.96 \\
$\mathrm{M}$ & 10.56 & 9.97 & 5.29 \\
& & & 4.67 & $t_{(2,618)}=.881$ \\
& & & .379 \\
\hline
\end{tabular}


Tabla 6

Media, desviación estándar y prueba ANOVA en las tres láminas del CREA según la edad de los adolescentes.

\begin{tabular}{|c|c|c|c|c|}
\hline Edad & & CREA A & CREA B & CREA C \\
\hline \multirow{2}{*}{$\begin{array}{l}12 \text { años } \\
(\mathrm{n}=71)\end{array}$} & $\mathrm{M}$ & 8.29 & 8.05 & 8.47 \\
\hline & $\mathrm{DE}$ & 2.96 & 3.03 & 3.59 \\
\hline \multirow{2}{*}{$\begin{array}{l}13 \text { años } \\
(\mathrm{n}=53)\end{array}$} & $\mathrm{M}$ & 9.58 & 9.01 & 10.67 \\
\hline & $\mathrm{DE}$ & 4.34 & 4.27 & 5.57 \\
\hline \multirow{2}{*}{$\begin{array}{l}14 \text { años } \\
(\mathrm{n}=89)\end{array}$} & M & 10.39 & 10.77 & 10.64 \\
\hline & $\mathrm{DE}$ & 4.63 & 5.23 & 6.18 \\
\hline \multirow{2}{*}{$\begin{array}{c}15 \text { años } \\
(\mathrm{n}=124)\end{array}$} & M & 11.24 & 11.26 & 10.26 \\
\hline & $\mathrm{DE}$ & 4.47 & 4.912 & 4.75 \\
\hline \multirow{2}{*}{$\begin{array}{c}16 \text { años } \\
(\mathrm{n}=119)\end{array}$} & M & 11.38 & 11.40 & 10.17 \\
\hline & $\mathrm{DE}$ & 5.12 & 5.13 & 6.24 \\
\hline \multirow{3}{*}{$\begin{array}{c}17 \text { años } \\
(\mathrm{n}=264)\end{array}$} & M & 11.94 & 11.52 & 10.36 \\
\hline & $\mathrm{DE}$ & 5.00 & 5.42 & 5.51 \\
\hline & $p$ & $\begin{array}{c}\mathrm{F}_{(5,714)}=8.38 \\
<.001\end{array}$ & $\begin{array}{c}\mathrm{F}_{(5,714)}=7.27 \\
<.001\end{array}$ & $\begin{array}{c}F_{(5,614)}=1.66 \\
.142\end{array}$ \\
\hline
\end{tabular}

Se realizaron contrastes post hoc y se observaron diferencias significativas en la Lámina A entre las medias de los adolescentes de 12 años y los de 14, 15, 16 y 17; entre los de 13 y los de 15, 16, 17 y entre los de 14 y los de 17. En la Lámina B se observaron diferencias entre los adolescentes de 12 años y los de 13, 14, 15, 16 y 17 y entre los de 13 años y los de 14, 15, 16 y 17. En la Lámina C se observaron diferencias entre los de 12 y los de 13,14, 15, 16 y 17 años.

Tabla 7

Media, desviación estándar y prueba $t$ en las tres láminas del CREA según dos grupos de edades: 12 - 14 años y 15 - 17 años.

\begin{tabular}{ccccc}
\hline Edad & & CREA A & CREA B & CREA C \\
\hline 12-14 años & M & 9.49 & 9.43 & 9.90 \\
& DE & 4.15 & 4.51 & 5.33 \\
$15-17$ años & M & 11.64 & 11.43 & 10.30 \\
& DE & 4.91 & 5.23 & 5.50 \\
& & & \\
& & $t_{(1,718)}=-5,59$ & $t_{(1,718)}=-4,87$ & $t_{(1,718)}=-, 86$ \\
& $p$ & $<.001$. & $<.001$ & .386 \\
\hline
\end{tabular}


Considerando las diferencias observadas, es pertinente presentar baremos según género y edad para el grupo de adolescentes. Se proponen a continuación percentiles para cada una de las láminas del CREA en varones y mujeres de 12 a 14 años y de 15 a 17 años (ver Tablas 8 y 9).

\section{Adultos}

Los puntajes medios hallados en la presente investigación (Lámina $\mathrm{A}: \mathrm{M}=11.40, \mathrm{DE}=5.11$; Lámina $\mathrm{B}: \mathrm{M}=10.72, \mathrm{DE}=4.91$; Lámina $\mathrm{C}: \mathrm{M}$ $=10.08, \mathrm{DE}=5.00)$ son similares a los establecidos en el manual del Test CREA para la población argentina adulta en la Lámina A $(\mathrm{M}=11.53, \mathrm{DE}$ $=5.3)$ y los valores superiores en la Lámina $\mathrm{B}$ $(\mathrm{M}=8.56, \mathrm{DE}=5.6)$. Con respecto a la incidencia del género en el CREA, se observaron difer encias estadísticamente significativas a favor de las mujeres en las Láminas B y C del CREA (ver Tabla 10). También se observaron diferencias en el CREA según la edad (ver Tabla 11) y la escolaridad (ver Tabla 12). Los análisis de varianza en los que se mantienen fijos los demás factores indican diferencias significativas entre los puntajes medios según la edad y el nivel de escolaridad en las tres Láminas del CREA.

Tabla 8

Percentiles en las tres láminas del CREA para adolescentes mujeres de 12 a 14 y de 15 a 17 años.

\begin{tabular}{ccccccc}
\hline & \multicolumn{3}{c}{$\begin{array}{c}\text { Adolescentes mujeres } \\
\text { de } \mathbf{1 2} \text { a } \mathbf{1 4}\end{array}$} & \multicolumn{3}{c}{$\begin{array}{c}\text { Adolescentes mujeres } \\
\text { de 15 a 17 }\end{array}$} \\
\hline Percentil & A & B & C & A & B & C \\
\hline 95 & 19 & 19 & 23 & 21 & 21 & 22 \\
90 & 15 & 15 & 16 & 18 & 20 & 18 \\
75 & 11 & 12 & 13 & 14 & 14 & 13 \\
50 & 9 & 8 & 9 & 10 & 10 & 9 \\
25 & 6 & 6 & 6 & 8 & 7 & 7
\end{tabular}

\begin{tabular}{ccccccc}
10 & 5 & 5 & 5 & 6 & 6 & 5 \\
5 & 4 & 4 & 4 & 5 & 5 & 4 \\
$\mathrm{~N}$ & 120 & 120 & 120 & 219 & 219 & 219 \\
$\mathrm{M}$ & 9.50 & 9.43 & 9.96 & 11.35 & 11.16 & 10.55 \\
$\mathrm{DE}$ & 4.51 & 4.72 & 5.58 & 4.85 & 5.14 & 5.56 \\
\hline
\end{tabular}

Tabla 9

Percentiles en las tres láminas del CREA para adolescentes varones de 12 a 14 y de 15 a 17 años.

\begin{tabular}{ccccccc}
\hline & \multicolumn{3}{c}{$\begin{array}{c}\text { Adolescentes varones } \\
\text { de 12 a 14 }\end{array}$} & \multicolumn{3}{c}{$\begin{array}{c}\text { Adolescentes varones } \\
\text { de 15 a 17 }\end{array}$} \\
\hline Percentil & A & B & C & A & B & C \\
\hline 95 & 16 & 18 & 20 & 20 & 21 & 21 \\
90 & 15 & 15 & 16 & 18 & 20 & 18 \\
75 & 12 & 12 & 12 & 15 & 14 & 13 \\
50 & 9 & 9 & 9 & 11 & 11 & 9 \\
25 & 7 & 6 & 6 & 8 & 8 & 6 \\
10 & 5 & 5 & 5 & 6 & 6 & 4 \\
5 & 4 & 4 & 4 & 5 & 5 & 3 \\
$\mathrm{~N}$ & 93 & 93 & 85 & 288 & 288 & 196 \\
$\mathrm{M}$ & 9.47 & 9.43 & 9.81 & 11.85 & 11.63 & 10.02 \\
$\mathrm{DE}$ & 3.66 & 4.23 & 4.99 & 4.95 & 5.29 & 5.42 \\
\hline
\end{tabular}

\section{Discusión}

Se han observado diferencias significativas en las puntuaciones medias del CREA según la variable edad en los tres grupos estudiados. En el caso de los adolescentes también se observaron diferencias según género y en los adultos según nivel de escolaridad. Se han propuesto datos normativos que atienden a la incidencia de las variables mencionadas. Los datos presentados pueden contribuir a una mejor interpretación de los resultados de grupos particulares. Asimismo, representan herramientas valiosas para el diagnóstico y el análisis de capacidades creativas individuales.

La edad de las personas es una variable importante para considerar en la evaluación de la 
creatividad en general y en el Test CREA. Investigaciones previas han señalado la necesidad de tener en cuenta la edad como variable de relevancia en la medición de la creatividad (Wechsler, 2006; Hang-Wu, Cheng, Man-Ip, \& McBride-Chang, 2005). También en el Test CREA se han observado diferencias según la edad de los participantes (Chacón-Araya \& Moncada-Jiménez, 2006; Donolo \& Elisondo, 2007; Elisondo \& Donolo, 2011; Limiñana-Gras et al., 2010; López-Martínez \& Navarro-Lozano, 2008).

En los análisis también se observaron diferencias según el nivel de escolaridad de los participantes. Estudios anteriores señalan diferencias según el nivel de escolaridad de los participantes en el CREA (Elisondo \& Donolo, 2011) y en otras pruebas de capacidades creativas (Matud, Rodríguez, \& Grande, 2007). La consideración del nivel educativo en la evaluación de la creatividad, específicamente en el Test CREA resulta indispensable considerando las notorias diferencias observadas entre grupos de diferente nivel de escolaridad. El análisis de los datos permite observar claramente que los puntajes ascienden conforme aumenta el nivel de escolaridad alcanzada por los sujetos. En las interpretaciones de las puntuaciones individuales se sugiere considerar el nivel de escolaridad de las personas, los datos normativos propuestos permiten interpretaciones mejor adaptadas a las condiciones particulares de las personas que resuelven el CREA.

Tabla 10

Media, desviación típica y prueba $t$ en las tres láminas del CREA según género para adultos.

\begin{tabular}{ccccc}
\hline Género & & A & B & C \\
\hline Mujeres & $\mathrm{M}$ & 11.55 & 11.16 & 10.39 \\
& $\mathrm{DE}$ & 5.07 & 4.96 & 4.98 \\
Varones & $\mathrm{M}$ & 11.17 & 9.99 & 9.61 \\
& $\mathrm{DE}$ & 5.16 & 4.74 & 5.01 \\
& & $t_{(1,1402)}=1.35$ & $t_{(1,2004)}=5.22$ & $t_{(1,1103)}=2.60$ \\
& & .177 & $<.001$ & .009 \\
\hline
\end{tabular}

Tabla 11

Media, desviación típica y ANOVA en las tres láminas del CREA según edad de los adultos.

\begin{tabular}{ccccc}
\hline Edad & & A & B & C \\
\hline $18-23$ años & $\mathrm{M}$ & 12.20 & 11.59 & 11.02 \\
$(\mathrm{n}=1096)$ & $\mathrm{DE}$ & 5.23 & 4.96 & 4.98 \\
$23-40$ años & $\mathrm{M}$ & 11.42 & 10.70 & 9.79 \\
$(\mathrm{n}=627)$ & $\mathrm{DE}$ & 4.85 & 4.92 & 4.88 \\
$41-55$ años & $\mathrm{M}$ & 9.69 & 9.00 & 7.84 \\
$(\mathrm{n}=308)$ & $\mathrm{DE}$ & 4.65 & 4.09 & 4.41 \\
56 años o más & $\mathrm{M}$ & 8.41 & 8.00 & 6.57 \\
$(\mathrm{n}=116)$ & $\mathrm{DE}$ & 4.89 & 4.18 & 4.07 \\
& $\mathrm{~F}$ & $\mathrm{~F}_{(3,1432)}=23.02^{* *}$ & $\mathrm{~F}_{(3,2032)}=36.47^{* *}$ & $\mathrm{~F}_{(3.1147)}=25.14^{* *}$ \\
\hline
\end{tabular}

Nota. $* * p<.01$ 
Se realizaron contrastes post hoc y se observaron diferencias significativas en las tres láminas entre todos los grupos etarios, excepto entre 41-55 y más de 56 años.

Tabla 12

Media desviación típica y ANOVA en las tres láminas del CREA según nivel de escolaridad de los adultos.

\begin{tabular}{ccccc}
\hline Escolaridad & & A & B & C \\
\hline Primario & $\mathrm{M}$ & 7.68 & 7.85 & 6.81 \\
& $\mathrm{DE}$ & 4.29 & 3.86 & 4.21 \\
Secundario & $\mathrm{M}$ & 9.77 & 9.11 & 8.43 \\
& $\mathrm{DE}$ & 4.46 & 4.12 & 4.44 \\
\multirow{3}{*}{ Superior } & $\mathrm{M}$ & 12.44 & 11.90 & 10.91 \\
& $\mathrm{DE}$ & 5.07 & 5.02 & 4.98 \\
& & $\mathrm{~F}_{(2.1435)}=78.41^{* *}$ & $\mathrm{~F}_{(2.2035)}=108.26^{* *}$ & $\mathrm{~F}_{(2.1150)}=47.27^{* *}$ \\
\hline
\end{tabular}

Nota. $* * p<.01$

Se realizaron contrastes post hoc en las tres láminas y entre los tres grupos se observaron diferencias significativas en los puntajes medios considerando el nivel de escolaridad alcanzado.

Considerando las diferencias observadas en el grupo de adultos, es necesario construir baremos diferentes según edad y escolaridad. Se presentan a continuación datos normativos para adultos de diferentes grupos etarios y niveles de escolaridad alcanzados (ver Tablas 13 y 14).

Tabla 13

Percentiles para adultos (varones y mujeres) según diferentes rangos de edades para las láminas A, B y C del CREA.

\begin{tabular}{cccccccccccccc}
\hline & & \multicolumn{1}{c}{$\mathbf{1 8} \mathbf{a} \mathbf{2 3}$} & $\mathbf{2 4 - 4 0}$ & \multicolumn{3}{c}{$\mathbf{4 1 - 5 5}$} & \multicolumn{3}{c}{ Más de 55 } \\
\hline & & $\mathbf{A}$ & $\mathbf{B}$ & $\mathbf{C}$ & $\mathbf{A}$ & $\mathbf{B}$ & $\mathbf{C}$ & $\mathbf{A}$ & $\mathbf{B}$ & $\mathbf{C}$ & $\mathbf{A}$ & $\mathbf{B}$ & $\mathbf{C}$ \\
\hline & 95 & 22 & 21 & 20 & 20 & 19 & 19 & 19 & 17 & 17 & 17 & 16 & 15 \\
& 90 & 19 & 18 & 18 & 18 & 17 & 17 & 16 & 14 & 13 & 13 & 13 & 12 \\
& 75 & 15 & 14 & 14 & 15 & 13 & 13 & 13 & 11 & 11 & 11 & 10 & 9 \\
& 50 & 11 & 11 & 10 & 11 & 10 & 9 & 9 & 8 & 7 & 8 & 7 & 7 \\
& 25 & 8 & 8 & 7 & 8 & 7 & 6 & 6 & 6 & 5 & 6 & 5 & 3 \\
& 10 & 6 & 6 & 6 & 6 & 6 & 4 & 4 & 4 & 3 & 1 & 4 & 1 \\
& 5 & 5 & 5 & 5 & 4 & 4 & 3 & 3 & 3 & 1 & - & 2 & - \\
$\mathrm{N}$ & & 707 & 987 & 592 & 453 & 627 & 385 & 202 & 308 & 129 & 76 & 116 & 47 \\
$\mathrm{M}$ & 12.20 & 11.58 & 11.02 & 11.41 & 10.70 & 9.79 & 9.68 & 9.00 & 7.84 & 8.40 & 8.00 & 6.57 \\
$\mathrm{DE}$ & 5.19 & 4.96 & 4.98 & 4.85 & 4.92 & 4.88 & 4.65 & 4.09 & 4.40 & 4.89 & 4.18 & 4.07 \\
\hline
\end{tabular}


Tabla 14

Percentiles para adultos (varones y mujeres) según los diferentes niveles de escolaridad para las láminas A, B y C del CREA.

\begin{tabular}{ccccccccccc}
\hline & & \multicolumn{3}{c}{ Nivel primario } & \multicolumn{3}{c}{ Nivel secundario } & \multicolumn{3}{c}{ Nivel superior } \\
\hline & Percentil & A & B & C & A & B & C & A & B & C \\
\hline & 95 & 16 & 15 & 15 & 18 & 17 & 17 & 22 & 21 & 20 \\
& 90 & 13 & 13 & 12 & 16 & 14 & 14 & 19 & 19 & 18 \\
& 75 & 10 & 10 & 9 & 12 & 11 & 11 & 15 & 15 & 14 \\
& 50 & 7 & 7 & 7 & 9 & 9 & 8 & 12 & 11 & 10 \\
& 25 & 5 & 6 & 4 & 7 & 6 & 5 & 9 & 8 & 7 \\
& 10 & 3 & 4 & 1 & 5 & 5 & 3 & 7 & 6 & 5 \\
& 5 & - & 2 & - & 4 & 4 & 1 & 5 & 5 & 4 \\
$\mathrm{~N}$ & & 127 & 171 & 93 & 333 & 615 & 232 & 978 & 1252 & 828 \\
$\mathrm{M}$ & & 7.67 & 7.85 & 6.80 & 9.77 & 9.11 & 8.42 & 12.43 & 11.89 & 10.90 \\
$\mathrm{DE}$ & & 4.29 & 3.86 & 4.21 & 4.46 & 4.12 & 4.44 & 5.04 & 5.01 & 4.98 \\
\hline
\end{tabular}

Con respecto a la incidencia de la variable género, se observaron algunas diferencias en los grupos de adolescentes y adultos. En el último grupo, cuando se analizó la incidencia del género en interrelación con la edad y la escolaridad no se observaron diferencias significativas. En estudios anteriores, se observaron resultados similares, es decir, inexistencia de diferencia según género en adultos (Chacón-Araya \& Moncada-Jiménez, 2006; Clapham \& King, 2010; Limiñana-Gras et al., 2010). Las investigaciones sobre creatividad y género muestran resultados diferentes según los grupos, los instrumentos que se utilizan y los contextos de análisis. Baer y Kaufman (2008) analizaron diferentes estudios sobre creatividad y género y concluyeron que los resultados de las investigaciones psicométricas referidas a diferencias de género son inconsistentes.

Los resultados hallados abren interesantes líneas para investigaciones futuras. Durante el proceso de recolección de datos, se han observado algunas dificultades en niños menores de 8 años para comprender la consigna del CREA. Es importante analizar, en futuros estudios, los procesos metacognitivos en los cuales se basa la tarea propuesta. Asimismo, se sugiere en futuras investigaciones analizar con detalle el proceso de administración del test CREA en niños registrando expresiones, comportamientos y dificultades durante la resolución de la tarea. En el grupo de los adultos, la edad también es una variable importante a considerar, especialmente en personas mayores con ciertos déficits motrices, visuales y auditivos que dificultan la realización de las tareas propuestas por el CREA, tal como se ha observado en el proceso de recolección de datos. Se propone para futuros estudios ajustar las pautas de administración e interpretación considerando la situación de los participantes y sus dificultades para resolver las tareas. Desarrollar diferentes estrategias de validación según el proceso de respuesta (entrevistas, observaciones participantes, grupos focales, protocolos verbales) es una promisoria línea de estudio del Test CREA.

Las experiencias escolares de las personas inciden en las posibilidades de resolver la tarea verbal y de escritura que propone el CREA, se recomienda en investigaciones posteriores considerar otras técnicas no verbales $\mathrm{u}$ otras estrategias para la evaluación integral de la creatividad 
en grupos con niveles bajos de escolaridad. En el caso de los adolescentes, las diferencias observadas según género abren interesantes líneas de investigaciones sobre creatividad en esta compleja etapa evolutiva. La realización de estudios mixtos con metodologías diversas puede ser de relevancia en la evaluación de la creatividad durante la adolescencia.

La conformación de la muestra y de los grupos según las variables consideradas es una limitante del estudio. En algunos casos, los datos normativos corresponden a grupos pequeños, como por ejemplo los de niños de 6 a 8 años en las Láminas A y B o los de adultos mayores de 55 años en la Lámina C. En futuras investigaciones es relevante ampliar el número de casos para estos grupos y construir datos normativos basados en muestras más numerosas y heterogéneas. La conformación de la muestra de manera no probabilística también representa una limitación del estudio y de los datos normativos presentados. Es necesario además, construir muestras representativas y ampliar los alcances de las mismas a otras regiones geográficas. Asimismo, en futuros estudios resulta de relevancia considerar variables como nivel socio-económico, contexto cultural, experiencias de ocio, etc. Sin embargo, se destaca como aporte la presentación de datos normativos que pueden contribuir en las interpretaciones de puntuaciones individuales y en el diagnóstico diferencial.

\section{Referencias}

Antoñanzas-Laborda, J. L., Toner-Pelay, M., Salavera-Bordás, C., Soler-Costa, R., \& Usan-Supervia, P. (2015). Creatividad y aprendizaje en niños de 4 y 5 años. Revista de Psicología y Educación, 10(1), 139-152. Recuperado de http://www.revistadepsicologiayeducacion.es
Aranguren, M. (2014). Validez de constructo del Test de Pensamiento Creativo de Torrance en una muestra de jóvenes argentinos. Anuario de Psicología/The UB Journal of Psychology, 44(1), 55-70. Recuperado de https://www.raco.cat/index.php/AnuarioPsicologia

Aranguren, M., \& Irrazabal, N. (2012). Diseño de una escala para la evaluación del comportamiento creativo en diferentes dominios. Ciencias Psicológicas, VI(1), 29-41. Recuperado de http://revistas.ucu.edu.uy/index.php/cienciaspsicologicas/index

Baer, J. (2012). Domain specificity and the limits of creativity theory. Journal of Creative Behavior, 46(1), 1629. doi: 10.1002/jocb.002

Baer, J., \& Kaufman, J. (2008). Gender differences in creativity. Journal of Creative Behavior, 42(2), 75-106. doi: 10.1002/j.2162-6057.2008.tb01289.x

Barbot, B., Besançon, M., \& Lubart, T. (2016). The generality-specificity of creativity: Exploring the structure of creative potential with EPoC. Learning and Individual Differences, 52, 178-187. doi: 10.1016/j. lindif.2016.06.005

Batey, M. (2007). A psychometric investigation of everyday creativity (Tesis doctoral inédita). University College, Londres.

Batey, M. (2012). The measurement of creativity: From definitional consensus to the introduction of a new heuristic framework. Creativity Research Journal, 24(1), 55-65. doi: 10.1080/10400419.2012.649181

Bogaert-García, H. (2017). La creatividad en los estudiantes universitarios. Una investigación psicométrica en la República Dominicana. Ciencia y Sociedad, 42(3), 51-68. doi: 10.22206/cys.2017.v42i3.pp51-68

Cárdenas-Avila, N., López-Fernández, V., \& Arias-Castro, C. (2018). Análisis de la relación entre creatividad, atención y rendimiento escolar en niños y niñas de más de 9 años en Colombia. Psicogente, 21(39), 7587. doi: 10.17081/psico.21.39.2823

Carson, S. H., Peterson, J. B., \& Higgins, D. M. (2005). Reliability, validity, and factor structure of the Creative Achievement Questionnaire. Creativi- 
ty Research Journal, 17(1), 37-50. doi: 10.1207/ s15326934crj1701_4

Castañeda-Rey, E. L., López, V., \& Ezquerro-Cordón, A. (2017). Creatividad, atención, rendimiento académico e interacción grupal en un aula de secundaria. Ulu: Revista científica sobre la imaginación, 2, 1-14. Recuperado de http://digibug.ugr.es/handle/10481/39268

Chacón-Araya, Y., \& Moncada-Jiménez, J. (2006). Relación entre personalidad y creatividad en estudiantes de educación física. Actualidades Investigativas en Educación, 6(1), 1-19. doi: 10.15517/aie.v6i1.9200

Chiecher, A. C., Elisondo, R. C., Paoloni, P. V., \& Donolo, D. S. (2018). Creatividad, género y rendimiento académico en ingresantes de ingeniería. Revista Iberoamericana de Educación Superior, 9(24), 138-151. Recuperado de https://www.ries.universia.unam.mx

Clapham, M. M., \& King, W. R. (2010). Psychometric characteristics of the CREA in an english speaking population. Anales de Psicología, 26(2), 206-211. Recuperado de http://revistas.um.es/analesps

Contini, E. N. (2013). Valorización y desvalorización de los tests en el campo de la psicología argentina. Una disociación no superada. PSIENCIA. Revista Latinoamericana de Ciencia Psicológica, 5(2), 119- 122. Recuperado de http://www.psiencia.org

Corbalán-Berná, J. (2008). ¿De qué se habla cuando hablamos de creatividad? Cuadernos FHyCSUNJu, 35, 11-21. Recuperado de http://www.scielo.org.ar

Corbalán-Berná, J., Martín-Brufau, R., Donolo, D., Clapham, M., Limiñana, R., García-Peñas, V., ... King, R. (2014). CREA. A cross-cultural study. Personality and Individual Differences, 60, S54. doi: 10.1016/j.paid.2013.07.223

Corbalán-Berná,, J., Martínez Zaragoza, F., Donolo, D. S., Alonso-Monreal, C., Tejerina-Arreal, M., \& Limiñana-Gras, M. (2003). CREA. Inteligencia Creativa. Una medida cognitiva de la creatividad. Madrid, España: TEA Ediciones.

Donolo, D. S., \& Elisondo, R.C. (2007). Creativity for all.
Consideration about a particular group. Annals of Psychology, 23(1), 148-151. Recuperado de http:// revistas.um.es/analesps

Elisondo, R. C. (2015). Evaluación de la creatividad. Análisis de variables alternativas relacionadas con la forma y el contenido de las respuestas en el CREA (Tesis doctoral). Universidad de Murcia, España. Recuperado de https://www.tdx.cat

Elisondo, R. C., \& Donolo, D. S. (2010). Creatividad o inteligencia. That is not the question. Anales de Psicología, 26(2), 220-225. Recuperado de http://revistas. um.es/analesps

Elisondo, R. C., \& Donolo, D. S. (2011). Los estímulos en un test de Creatividad. Incidencias según género, edad y escolaridad. Boletín de Psicología, 101, 5165. Recuperado de https://www.uv.es/seoane/boletin/boletin.html

Elisondo, R. C., \& Donolo, D. S. (2014). Interculturalidad, apertura a experiencias y creatividad Aportes para una educación alternativa. Red, Revista de Educación a Distancia, 41, 1-19. Recuperado de https:// www.um.es/ead/red

Elisondo, R. C., \& Donolo, D. S. (2016a). Construcción y análisis de las propiedades psicométricas del Cuestionario de Acciones Creativas en población argentina. PSIENCIA, Revista Latinoamericana de Ciencia Psicológica, 8(1), 1-21. Recuperado de http://www. psiencia.org

Elisondo, R. C., \& Donolo, D. S. (2016b). Creativity, involvement, and leisure time: The more, the better. Business Creativity and the Creative Economy, 2(1), 102-109. Recuperado de http://www.icscpress.com/ journals/bcce

Elisondo, R. C., Donolo, D. S., \& Corbalán-Berná, F. J. (2009). Evaluación de la Creatividad ¿Relaciones con inteligencia y personalidad? Revista Iberoamericana de Diagnóstico y Evaluación Psicológica, 28(2), 67-79. Recuperado de http://www.aidep.org/ numeros-publicados

Elisondo, R. C, Donolo, D. S., \& Limiñana-Gras, R. M. 
(2018). The measure of originality in CREA test responses. Anales de Psicología/Annals of Psychology, 34(1), 197-210. doi: 10.6018/analesps.34.1.286131

Freiberg-Hoffmann, A., \& Fernández-Liporace, M. (2017). Graphic Creativity Assessment: Psychometric properties in college students from Buenos Aires. Psychological Thought, 10(1), 60-79. doi: 10.5964/ psyct.v10i1.192

Guilford, J. P. (1962). Creativity: it's measurement and development. En S. Parnes \& H. Harding (Eds.), A source book for creative thinking. New York: Scribner's.

Hang-Wu, C., Cheng, Y., Man-Ip, H., \& McBride-Chang, C. (2005). Age differences in creativity: Task structure and knowledge base. Creativity Research Journal, 17(4), 321-326. doi: 10.1207/s15326934crj1704_3

Hocevar, D. (abril, 1979). The development of the Creative Behavior Inventory (CBI). Trabajo presentado en el encuentro anual Rocky Mountain Psychological Association. Recuperado de https://eric.ed.gov/?i$\mathrm{d}=\mathrm{ED} 170350$

Humble, S., Dixon, P., \& Mpofu, E. (2017). Factor structure of the Torrance Tests of Creative Thinking Figural Form A in Kiswahili speaking children: Multidimensionality and influences on creative behavior. Thinking Skills and Creativity, 27, 33-44. doi: 10.1016/j. tsc. 2017.11 .005

Krumm, G., Aranguren, M., Arán-Filippetti, V., \& Lemos, V. (2016). Factor structure of the Torrance Tests of Creative Thinking Verbal Form B in a Spanish speaking population. Journal of Creative Behavior, 50(2), 150-164. doi: 10.1002/jocb.76

Limiñana-Gras, R. M., Bordoy, M., Juste-Ballesta, G., \& Corbalán-Berná,, J. (2010). Creativity, intelectual abilities and response styles: Implications for academic performance in the secondary school. Anales de Psicología, 26(2), 212-219. Recuperado de http:// revistas.um.es/analesps

Long, H. (2014). An empirical review of research methodologies and methods in creativity studies (2003-2012). Creativity Research Journal, 26(4), 427-438. doi:

\subsection{0/10400419.2014.961781}

López-Martínez, O., \& Brufau, R. (2010). Creative intelligence and thinking styles. Annals of Psychology, 26(2), 254-258.

López-Martínez, O., \& Navarro-Lozano, J. (2008). Estudio comparativo entre medidas de creatividad: TTCT vs. CREA. Anales de Psicología, 24(1), 138-142. Recuperado de http://revistas.um.es/analesps

Martínez-Zaragoza, F. A. (2003). Características psicométricas del CREA (inteligencia creativa). Un estudio con población española y argentina. RIDEP, 16(2), 71-83. Recuperado de http://www.aidep.org

Matud, M., Rodríguez, C., \& J. Grande (2007). Gender differences in creative thinking. Personality and Individual Differences, 43(5), 1137-1147. doi: 10.1016/j. paid.2007.03.006

Primi, R., Nakano, T. de C., Morais, M. de F., Silva-Almeida, L. , \& Marques-David, A. P. (2013). Factorial structure analysis of the Torrance Test with Portuguese students. Estudos de Psicologia (Campinas), 30(1), 19-28. doi: 10.1590/s0103-166x2013000100003

Ramos-Moreno, A. M., López-Fernández, V., \& Llamas-Salguero, F. (2017). Relación entre la creatividad, la memoria inmediata y lógica en relación con el rendimiento académico en la educación secundaria. Revista Academia y Virtualidad, 10(1), 123-130. doi: 10.18359/ravi.2674

Richards, R. E. (2007). Everyday creativity and new views of human nature: Psychological, social, and spiritual perspectives. Washington, DC: American Psychological Association. doi: 10.1037/11595-000

Rodríguez-Suárez, P. A., Llamas-Salguero, F., \& López-Fernández, V. L. (2015). Relación entre la creatividad e inteligencia emocional en alumnado que cursa prácticas de Ingeniería en Colombia. ReiDoCrea: Revista Electrónica de Investigación y Docencia Creativa, 4, 192-199. Recuperado de http://digibug.ugr.es/handle/10481/21881

Said-Metwaly, S., Van den Noortgate, W., \& Kyndt, E. (2017). Methodological issues in measuring creativity: A systematic literature review. Creativity. Theo- 
ries - Research - Applications, 4(2), 276-301. doi:

10.1515/ctra-2017-0014

Sánchez-Hernández, O., Méndez, F. X., \& Garber, J. (2015).

Producción divergente explicativa: La relación entre

resiliencia y creatividad. Electronic Journal of Research in Educational Psychology, 13(37), 551-568. doi: 10.14204/ejrep.37.14126

Torrance, E. P. (1990). The Torrance Tests of Creative Thinking: Norms Technical manual figural (streamlined) forms $A \& B$. Bensenville, IL: Scholastic Testing Service Inc.

Wechsler, S. (2006). Validity of the Torrance Tests of Creative Thinking to the Brazilian culture. Creativity Research Journal, 18(1), 15-25. doi: 10.1207/ s15326934crj1801_3

Yarbrough, N. D. (2016). Assessment of creative thinking across cultures using the Torrance Tests of Creative Thinking (TTCT): Translation and validity issues. Creativity Research Journal, 28(2), 154-164. doi: 10.1080/10400419.2016.1162571

Yoon, C. H. (2017). A validation study of the Torrance Tests of Creative Thinking with a sample of Korean elementary school students. Thinking Skills and Creativity, 26, 38-50. doi: 10.1016/j.tsc.2017.05.004 